\title{
Device Grouping for Fast and Efficient Channel Access in IEEE 802.11ah based IoT Networks
}

\author{
Sabin Bhandari, Shree Krishna Sharma and Xianbin Wang \\ Department of Electrical and Computer Engineering, Western University, London, Ontario, Canada \\ Email: \{sbhanda7, sshar323, xianbin.wang\}@uwo.ca
}

\begin{abstract}
The recent advances in Internet of Things (IoT) have led to numerous emerging applications ranging from eHealthcare to industrial control, which often demand stringent Quality of Service (QoS) requirements such as low-latency and high system reliability. However, the ever-increasing number of connected devices in ultra-dense IoT networks and the dynamic traffic patterns increase the channel access delay and packet collision rate. In this regard, this paper proposes a sectorbased device grouping scheme for fast and efficient channel access in IEEE 802.11ah based IoT networks such that the total number of the connected devices within each sector is dramatically reduced. In the proposed framework, the Access Point (AP) divides its coverage area into different sectors, and then each sector is further divided into distinct groups based on the number of devices and their location information available from the cloud-center. Subsequently, individual groups within a sector are assigned to specific Random Access Window (RAW) slots, and the devices within distinct groups in different sectors access the allocated RAW slots by employing a spatial orthogonal access mechanism. The performance of the proposed sectorized device grouping scheme has been analyzed in terms of system delay and network throughput. Our simulation results show that the proposed scheme can significantly enhance the network throughput while simultaneously decreasing the system delay as compared to the conventional Distributed Coordination Function (DCF) and IEEE 802.11ah grouping scheme.
\end{abstract}

\section{INTRODUCTION}

The emerging Internet of Things (IoT) paradigm is expected to interconnect various objects and processes for massive information collection, analysis and utilization [1]. Many industries are putting significant amount of efforts in creating novel business models, products and services based on IoT platforms towards bringing economic and social benefits in various sectors such as industrial automation, health care, and transportation [2]. Consequently, the total number of connected sensors and machine type communication devices has been rapidly increasing over the recent years, and IHS Markit has predicted about 125 billion connected devices by 2030. However, due to limited radio resources available to support these massive number of connections and cost issues, the upcoming fifth generation $(5 \mathrm{G})$ wireless networks are expected to support extremely high device density up to about 1 million devices per square kilometers [3], thus leading to ultra-dense wireless IoT networks.

\section{A. Challenges in Ultra-Dense IoT Networks}

In ultra-dense IoT application scenarios such as industrial automation/control systems, providing fast channel access, and reliable and low-latency communication links becomes extremely challenging due to inefficient channel access mechanism, resource-constrained edge devices and limited available radio resources [4]. Due to contention-based nature of the most existing channel access schemes in unlicensed wireless networks, the problem of access network congestion becomes severe in ultra-dense IoT networks since the collision rate increases dramatically with the device density. Although the traditional IEEE 802.11 standard works well for the small local wireless network with a single Access Point (AP) supporting a reasonably small number of devices [5], scalability becomes the main issue in ultra-dense networks due to significant increase in the channel access delay and packet collision rate. Another major problem in ultra-dense networks comes from the device heterogeneity since the network has to support diverse Quality of Service (QoS) requirements of various IoT services [6]. One of the promising approaches to address this issue is to employ a suitable device grouping mechanism by enabling the efficient exchange of information among the network entities. To this end, this paper focuses on improving the efficiency of channel utilization in terms of system delay and throughput by employing a device grouping mechanism.

\section{B. Related Works}

The emergence of IoT has changed the perspective of wireless communications since the number of devices has exponentially increased over the recent years. In order to support these massive number of devices in the existing networks, the IEEE Task Group ah (TGah) is dedicated to the standardization of a new IEEE 802.11 ah protocol, which is customized for the large-scale networks [7]. The IEEE 802.11ah is an emerging wireless standard in sub- $1 \mathrm{GHz}$ license exempt bands for cost effective and range-extended communication. This standard adopts the grouping-based Medium Access Control (MAC) protocol to reduce the contention overhead [8]. Moreover, by utilizing the Multiple Input Multiple Output (MIMO) scheme, the IEEE 802.11ah based systems can utilize the benefits of spatial diversity to enhance the link capacity and to extend the coverage area [7].

Although the grouping based MAC protocol in IEEE 802.11ah standard significantly reduces the number of collisions and the contention overhead, existing solutions consider the grouping of devices/stations by using simplistic approaches either in a random or an uniform manner. In $[9,10]$, the authors proposed an optimal group division and resource allocation 
strategy for static network conditions. For the group based contention mechanism, the size of a group is the key design parameter since the number of stations significantly affects the network performance. However, the IEEE 802.11ah standard does not provide any guidelines for the group size. In this regard, the authors in [11] provided an expression to find the optimum group size on the basis of the number of active stations per group, traffic arrival rate, and the beacon interval. Nevertheless, in most of the existing solutions, devices are randomly assigned to different groups, and less attention has been given to the formation of efficient and reliable groups.

\section{Contributions}

In this paper, we propose to employ a sectorized grouping scheme in IEEE 802.11ah based ultra-dense IoT networks by employing a spatial orthogonal access scheme. The main objectives of the proposed sectorized grouping scheme are to reduce the channel contention by reducing the number of stations within a sector, to enable the spatial sharing of RAW slots among the overlapping APs/other neighbor stations in different groups, and to minimize the hidden station problem [12]. In the proposed scheme, the cloud-center provides the stations' information to the AP via an Internet link since it has a global knowledge of the network. The AP then broadcasts beacons to different geographical locations by utilizing simple sectorized beams. The number of stations is further divided into different groups uniformly within the sectors [8]. Thus divided groups are assigned to different RAW slots for the data transmission and they spatially access the channel for data transmission towards the AP. By considering this setup, the performance of the proposed sectorized grouping scheme is analyzed in terms of system delay and network throughput. Finally, the performance of the proposed scheme is compared with the conventional Distributed Coordination Function (DCF) and IEEE 802.11ah without sectorization via numerical results.

The rest of this paper is organized as follows. In Section II, a breif description of IEEE 802.11ah and its grouping mechanism are presented. In Section III, the overall system model and the proposed sector-based grouping scheme are described. In Section IV, the probability of transmission, throughput, and delay analysis of the proposed method are presented. In Section V, the performance of proposed scheme is evaluated via simulations. Finally, the paper is concluded in Section VI.

\section{GRouping Scheme IN IEEE 802.11 Ah STANDARD}

The IEEE 802.11ah standard is designed to support the applications with a large number of communicating devices, extended coverage area, and low energy consumption [13]. This new standard maintains similar network architecture as IEEE 802.11 for fixed, outdoor and point-to-multi-point applications. In order to meet the above mentioned requirements, IEEE 802.11ah differs from the traditional IEEE 802.11 in both the physical (PHY) and MAC layers. The PHY layer of IEEE 802.11ah is based on the IEEE 802.11ac and is a tenfold down-clocked version of IEEE 802.11ac, operating over a set

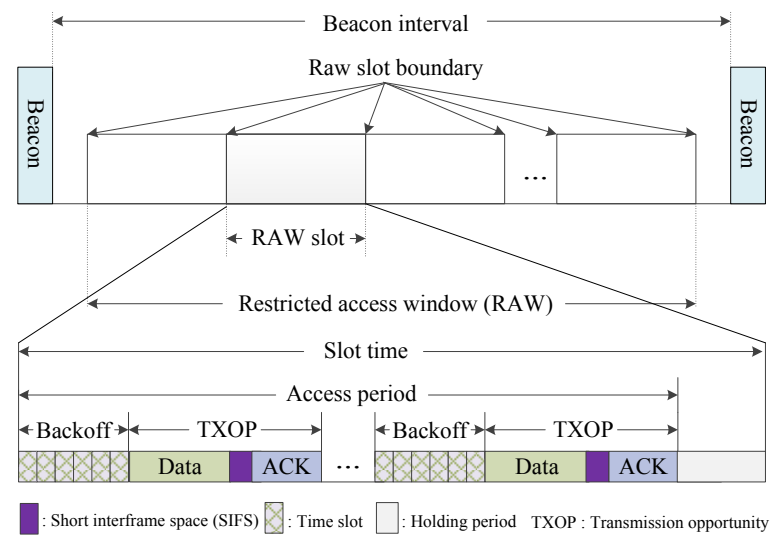

Fig. 1. Grouping-based MAC protocol in IEEE 802.11ah standard.

of unlicensed radio bands (all sub-1 GHz) [14]. A singleuser beamforming, MIMO, and downlink multi-user MIMO first presented in IEEE 802.11ac are also adopted in the IEEE 802.11ah standard [7].

In order to reduce the contention level in M2M networks with the thousands of devices, IEEE 802.11 ah has introduced a new grouping based MAC protocol. This protocol also supports the advanced power saving mechanism, improved medium access, and throughput enhancement [15]. In legacy IEEE 802.11 networks, the AP can support only up to 2008 stations. In addition, the Traffic Indication Map (TIM) bitmap imposes another limitation. However, the TGah has extended the range of Association ID (AID) numbers from 0-8191, and the length of TIM is also increased to 8192 bits to support a large number of stations [16]. In particular, sensors in a wireless network are partitioned into several groups. The channel access time is partitioned into beacon intervals, each of which is further divided into a number of equal duration RAW slots as depicted in Fig. 1. Each RAW slot is then assigned to a group of sensors, and only the devices within a particular group are allowed to access the RAW slot assigned to that group. Since only a part of stations contend for the channel access in a particular RAW slot, the collision probability becomes significantly lower as compared to the conventional IEEE 802.11ah.

\section{System Model ANd Proposed Grouping MECHANISM}

The future of networking is not only bounded with people but also is related to the integration of all objects, media and services, thus creating the Internet of Everything (IoE) [17]. The IoE connects the people, communicating objects or things, processes, and data in an effective manner to provide the ubiquitous services [18]. In this regard, we consider a large-scale IoT scenario composed of $N$ number of stations deployed over a circular area of radius $1 \mathrm{~km}$ as depicted in Fig. 2. The IoT network is organized as a hierarchical model, in which the AP is responsible to collect the information sensed by different stations/sensors. The data packets from each station are delivered to the AP in one hop. The gateway is then responsible for transferring the collected information to the 


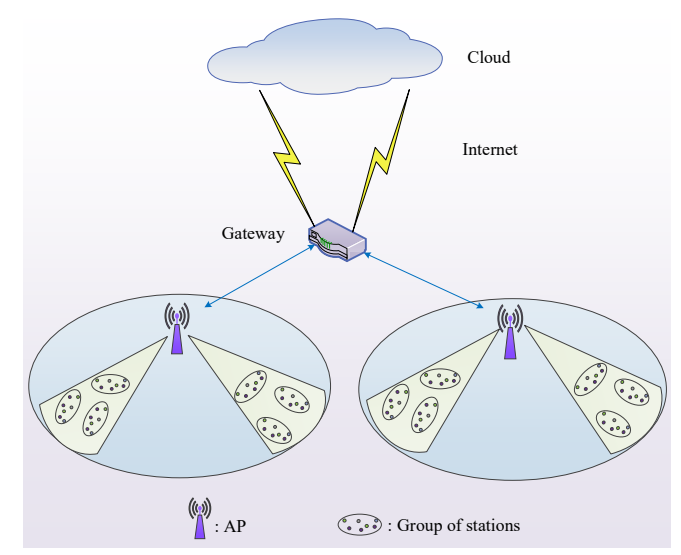

Fig. 2. Pictorial representation of the proposed grouping mechanism.

core network or cloud via an Internet link. Similarly, the cloudcenter provides the location information of each station to the AP. The gateway and AP are considered to be positioned at the specific geographic locations [19]. The pictorial representation of the proposed sector-based grouping scheme is shown in Fig. 2. In this scheme, the AP broadcasts beacons to the specific locations by utilizing the sectorized beams created with multiple antennas at the AP. The stations thus can take the advantages of distinct geographical areas since the AP coverage area is divided into different sectors.

In the considered scenario, the channel access time is divided into different mini-slots. We consider $K$ groups of stations, each with the size of $g_{k}$ and $\sum_{k=1}^{K} g_{k}=N, k=$ $1,2,3, \ldots, K$. Figure 2 shows the geographically sectorized areas of the APs using multiple beamforming antenna arrays. The data transmission from the stations in one sector to the AP does not interfere with the transmission in other sectors. Let $\varkappa$ be the number of stations in the $k$ th group of a particular sector. The sectorized beam of the AP covers different groups of size $g_{k}$. For the given station $s_{i, k}, i=1,2,3, \ldots, \varkappa$ in group $k$ of the given sector within a single AP, we can write the following relation as in [12]

$$
\sum_{i=1}^{\varkappa} s_{i, k} \ll \sum_{\forall k \in A P} g_{k} .
$$

The frame durations of a RAW and a RAW slot are denoted as $\Gamma_{r}$ and $\Gamma_{s}$, respectively. Similarly, $\Gamma_{s, k}$ denotes the time duration of the RAW slot allocated to the $k$ th group, and therefore, $\Gamma_{r}=\sum_{k=1}^{K} \Gamma_{s, k}$. The stations in a specific group periodically access the channel in the specified RAW slots. The proposed group formation procedure is detailed later in Algorithm 1 (Section IV).

\section{Probability of Transmission, Throughrut and DELAY ANALYSIS}

In this section, we present the probability of transmission, throughput and delay analysis of IEEE 802.11ah RAW for both the conventional DCF and our proposed approach. The DCF defines two medium access mechanisms to transmit the data packets, i.e., the basic access and Request-To-Send
(RTS)/Clear-To-Send (CTS). A station with the data packet senses the channel activity before transmitting towards the AP. If the channel is idle, a station transmits a packet immediately. However, if the channel is busy, the station persists to monitor the channel until it becomes idle for more than DCF Interframe Space (DIFS). To minimize the collision probability, the station waits until the random backoff interval before transmitting a packet. In the case of RTS/CTS, the short RTS and CTS packets are exchanged to reserve the channel prior to the data packet transmission. The neighboring stations will refrain the data packet transmission until the ongoing transmission is complete. More specifically, the RTS/CTS mechanism minimizes the data collision probability, collision duration, and also copes with hidden stations, and thus is more advantageous for ultra-dense IoT networks [20].

Let $n$ be the number of contending stations for the medium access in the given RAW slot. In the case of saturation conditions, each station immediately transfers the packet after the completion of each successful transmission. However, due to the consecutive transmissions, each packet needs to wait for the random backoff interval before transmitting. Let us assume that, at each transmission attempt, each packet collides with a constant and independent probability $p$. Moreover, when the number of stations tries to access the slot, devices are contended by the random back off procedure. Let $m$ and $C W_{\min }$ be the maximum back-off stage and minimum value of contention window, respectively. Then, the maximum value of contention window becomes $C W_{\max }=2^{m} C W_{\min }$ [21]. The transmission occurs only when the back-off counter becomes zero. The probability that a station transmits a packet in a randomly chosen slot is expressed as [21]

$$
P_{\tau}=\frac{2(1-2 p)}{(1-2 p)\left(C W_{\min }+1\right)+p C W_{\min }\left(1-(2 p)^{m}\right)} .
$$

Similarly, the conditional collision probability $p$ when a station transmits a packet is given by

$$
p=1-\left(1-P_{\tau}\right)^{(n-1)} .
$$

Subsequently, the probability $P_{t r}$ that there is at least one ongoing transmission in the considered time slot is calculated as

$$
P_{t r}=1-\left(1-P_{\tau}\right)^{n} \text {. }
$$

Next, the probability $P_{s}$ that a packet transmission occurring on a channel becomes successful is expressed as

$$
P_{s}=\frac{n P_{\tau}\left(1-P_{\tau}\right)^{(n-1)}}{1-\left(1-P_{\tau}\right)^{n}}
$$

The normalized system throughput is defined as the fraction of time that a random access channel is used to successfully transmit the payload bits [22], and can be expressed as

$$
S_{c}=\frac{P_{s} P_{t r} E\left[P_{l d}\right]}{\left(1-P_{t r}\right) \sigma+P_{t r} P_{s} T_{s}+P_{t r}\left(1-P_{s}\right) T_{c}},
$$

where $E\left[P_{l d}\right]$ is the average payload size, $T_{s}$ is the average time slots of successful transmission, $T_{c}$ is the average time slots of an unsuccessful transmission, and $\sigma$ is the backoff 
slot duration, respectively. The values of $T_{s}$ and $T_{c}$ can be calculated as [22]

$$
\begin{aligned}
& \begin{aligned}
& T_{s}= T_{R T S}+T_{C T S}+T_{P H Y_{h d r}}+T_{M A C_{h d r}}+E\left[P_{l d}\right]+ \\
& T_{A C K}+3 T_{S I F S}+T_{D I F S}+4 \delta,
\end{aligned} \\
& T_{c}=T_{R T S}+T_{D I F S}+\delta,
\end{aligned}
$$

where $T_{R T S}, T_{C T S}, T_{P H Y_{h d r}}, T_{M A C_{h d r}}$, and $T_{A C K}$ are the transmission times of RTS, CTS, PHY header, MAC header, and ACK frame, respectively. The $T_{S I F S}$ and $T_{D I F S}$ define the short and distributed inter-frame space durations, and $\delta$ is the propagation delay.

The above analysis is for the conventional DCF. Based on conventional DCF, the authors in [15] proposed centralized uniform and decentralized random grouping schemes without considering the geographical locations of stations. The conventional DCF is a distributed channel access mechanism for the standard WLAN to provide the long-term fair channel access for the stations. However, dense IoT stations may experience unfair channel allocations on the basis of their locations because of the propagation characteristic of radio signals, such as capture effect and path attenuation. The stations situated nearby an AP can access the channel several times more than a station located far from the AP. The authors in [21] assume that the collision probability $p$ is identical for all the stations. Consequently, the probability $P_{\tau}$ that a station transmits a packet in a given idle slot is also same for the all stations. However, in the realistic scenario, the probability of collision $p$ also depends on the distance from the AP. The stations far from the AP experiences high collisions as compared to the stations located nearby the AP. Thus a station located nearby an AP favorably transmits more data packets as compared to the station that is far from the AP. To address this aspect, our analysis incorporates the location of stations and the effect of the AP coverage sectorization.

The proposed grouping methodology and channel access mechanism is presented in Algorithm 1. In the proposed sectorized grouping scheme, the stations in the same sector can hear each other's transmission, and thus the hidden station problem is minimized [13]. Moreover, due to the sectorized beamforming, a large number of packets can be transmitted successfully towards the AP due to the lower packet collision rate. Hence, the throughput presented in (6) for the sectorized scenario can be expressed as [12]

$$
S_{c, \text { sector }}=\frac{P_{s} P_{t r} E\left[P_{l d}\right]}{\left(1-P_{t r}\right) \sigma+P_{t r} P_{s} T_{s}+P_{t r}\left(1-P_{s}\right) \zeta},
$$

where $\zeta$ is the residual frame collision coefficient. The AP coverage sectorization reduces the frame collision probability, and the coefficient $\zeta$ can be expressed as

$$
\zeta=p_{c_{k}} T_{c}
$$

The frame collision probability $p_{c_{k}}$ can be derived as [12]

$$
p_{c_{k}}=\left(1-\left(1-p_{\eta}\right)^{\vartheta(n-1)}\right),
$$

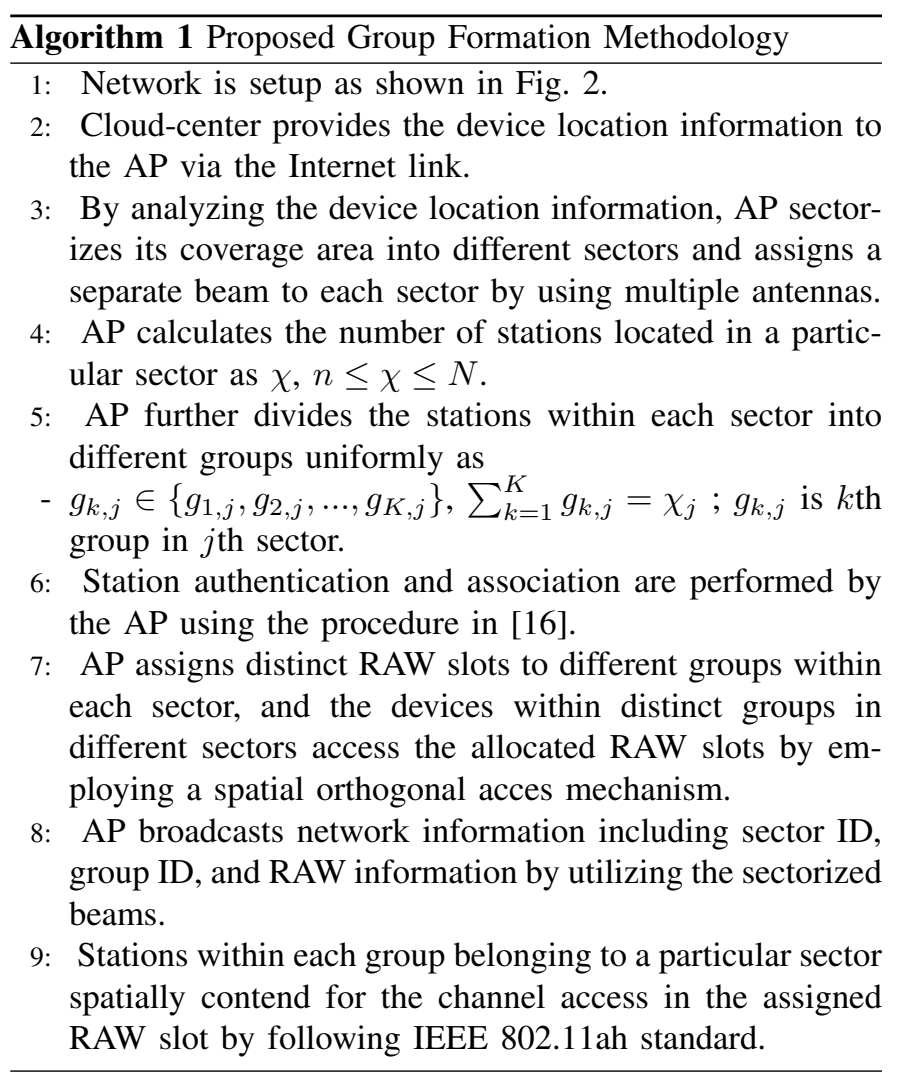

with

$$
p_{\eta}=1-\left(1-\frac{1}{C W_{\text {avg }}}\right)^{n-1},
$$

where $\vartheta$ is the slot time and $C W_{\text {avg }}$ is the average backoff window size.

The IEEE 802.11ah adopts the RAW scheme in order to cope with the packet collision, channel contention and the hidden station problem. A station is expected to contend for channel access during the allocated RAW slot. However, the stations belonging to the specified RAW slot may not hear each other's transmission in the ultra-dense IoT network, thus packet collision rate increases significantly. The sectorization involves the partitioning of the AP coverage area into different non-overlapping sectors by using antenna arrays. The frame collision coefficient due to the sectorized beam forming is given by (10), and the corresponding throughput can be calculated by substituting $T_{c}$ with $\zeta$ in (6) and is expressed in (9). As presented in Algorithm 1, the AP further divides the stations of the each sector into different groups uniformly. As a consequence, the stations belonging to a specified group within a sector can only gain the channel access during the allocated RAW slot, thus minimizing the frame collision probability given by (3) as compared to the conventional standard.

While analyzing the delay aspects, the average delay $E[D]$ for successfully transmitted data packet is given by [20]

$$
E[D]=E[X] E[\text { slot }],
$$

where $E[X]$ is the average number of time slot, and $E[$ slot $]$ 
TABLE I

NETWORK PARAMETERS

\begin{tabular}{cc}
\hline Parameters & Value \\
\hline Payload & 256 bytes \\
PHY Header & 128 bits \\
MAC Header & 272 bits \\
RTS Frame & PHY Header +160 bits \\
CTS Frame & PHY Header +112 bits \\
ACK Frame & PHY Header +112 bits \\
Time Slot & $50 \mu \mathrm{s}$ \\
SIFS & $28 \mu \mathrm{s}$ \\
DIFS & $128 \mu \mathrm{s}$ \\
$C W_{m i n}$ & 32 \\
$p_{c_{k}}$ & 0.01 \\
Propagation Delay & $1 \mu \mathrm{s}$ \\
Number of Sectors & 4 \\
\hline
\end{tabular}

is the average length of a slot time for the successful data transmission. The term $E[X]$ can be calculated as [20]

$$
E[X]=\frac{(1-2 p)\left(C W_{\min }+1\right)+p C W_{\min }\left(1-(2 p)^{m}\right)}{2(1-2 p)(1-p)} .
$$

\section{PERFormance AnAlysis}

In this section, we analyze and evaluate the performance of the proposed sectorized and grouping scheme in terms of the throughput and system delay by using the MATLAB software. The simulation parameters are presented in Table I.

\section{A. Throughput}

Figure 3 presents the normalized throughput of the proposed and conventional schemes versus the number of stations up to 1000 . Similarly, Fig. 4 shows the performance comparison of the proposed priority scheme with the conventional DCF and IEEE 802.11ah for the ultra-dense IoT scenario, i.e., the number of stations up to 4000 . In both the cases, the normalized throughput decreases as the number of stations increases because of the channel congestion and packet collisions. The DCF scheme is heavily influenced by the increasing number of hidden stations and packet collisions, which reduces the network throughput significantly. However, the throughput of IEEE 802.11ah is improved by reducing the

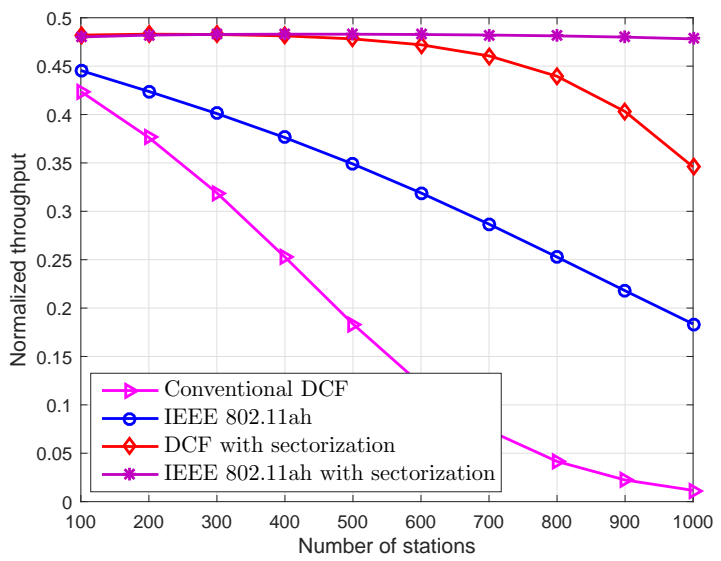

Fig. 3. Performance comparison of the proposed sectorized grouping scheme with conventional DCF and IEEE 802.11ah mechanism in terms of the normalized throughput.

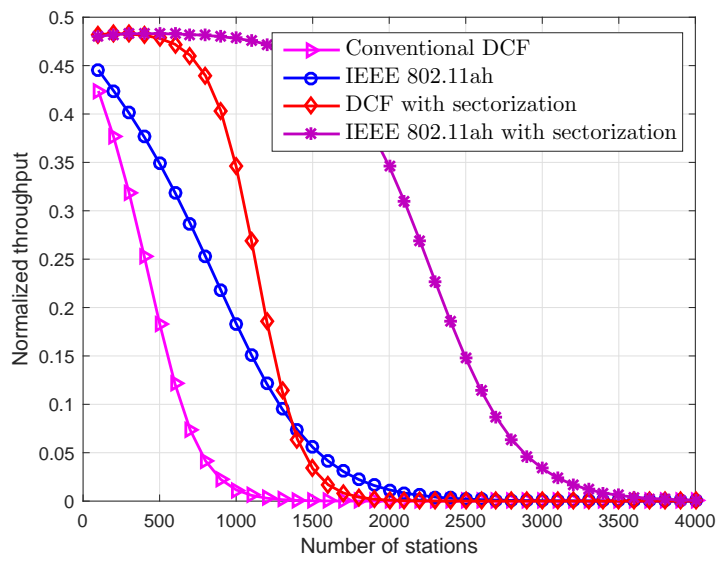

Fig. 4. Performance comparison of the proposed sectorized grouping scheme with conventional DCF and IEEE 802.11ah mechanism in terms of the throughput for ultra-dense IoT scenario (i.e., number of stations up to 4000).

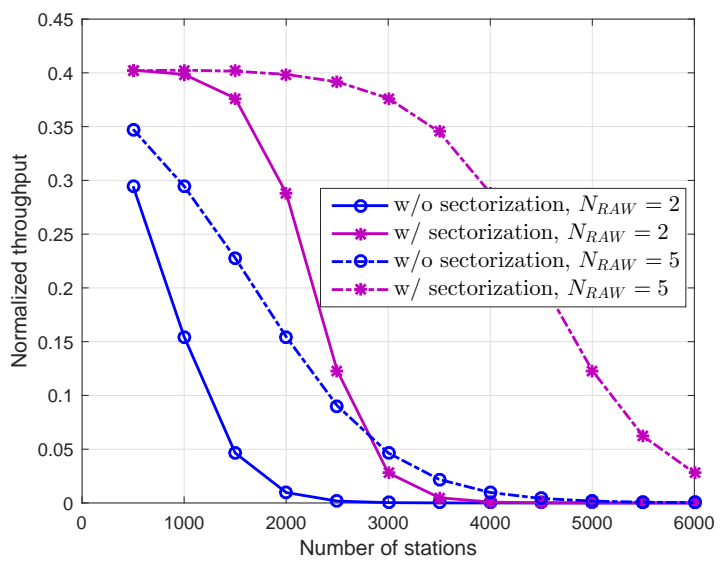

Fig. 5. The effect of number of RAW slots in throughput of the IEEE 802.11ah with and without sectorized grouping scheme.

number of stations in each group. Furthermore, the grouping based protocol decreases both the collision rate and the hidden terminals significantly. In addition, the sectorization of the AP coverage area further reduces the hidden stations and packet collisions by utilizing the distinct areas and simple sectorized beamforming. As a result, the proposed sector-based grouping scheme provides a significant throughput improvement by employing a spatially orthogonal access scheme.

Figure 5 shows the effect of the number of RAW slots $\left(N_{R A W}\right)$ in throughput performance of the proposed sectorized grouping scheme and IEEE 802.11ah. It has been noted that the throughput of the overall network is enhanced while increasing the value of $N_{R A W}$ from 2 to 5 . The number of RAW slots allows to limit the number of contending stations in a given interval of the time. However, relatively higher value of $N_{R A W}$ in IEEE 802.11ah is not enough to deliver the packets successfully because of the shorter time slot. In the proposed sectorized grouping scheme, stations within different groups can access the distinct orthogonal wireless channels with the reduced collision probability. 


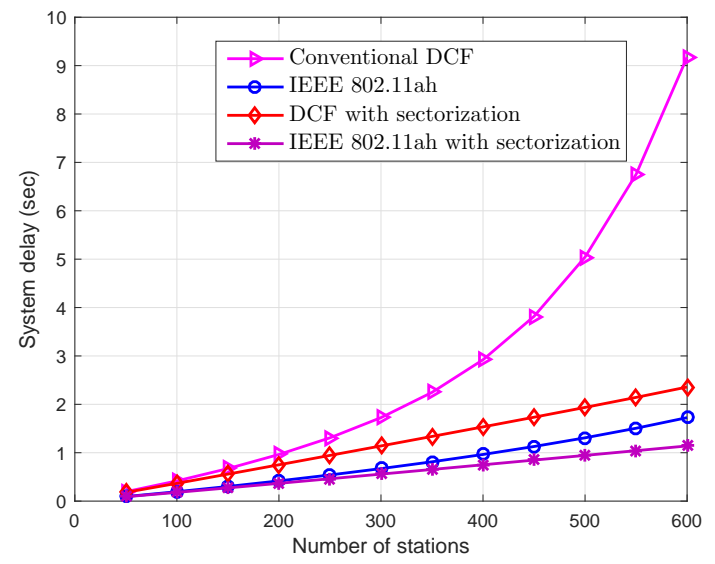

Fig. 6. Performance comparison of the proposed sectorized grouping scheme with conventional DCF and IEEE 802.11ah mechanism in terms of the system delay.

\section{B. System Delay}

Figure 6 depicts the overall system delay of the network versus the number of stations. It is clearly observed that the system delay increases as the number of stations increases. The intuitive reason behind this trend is the following. Each station in the network begins to experience the congestion problems because of a large number of stations. The number of packet collisions becomes more frequent, and the data retransmissions are more recurrent. The probability of packet loss also increases due to the link constraints, the retry limits, and collisions.

Also, from Fig. 6, it can be depicted that the conventional DCF scheme has the higher delay as compared to the IEEE 802.11 ah and the proposed scheme. In addition, the proposed sectorized grouping mechanism enhances the delay performance of IEEE 802.11ah. This performance gain is achieved due to the reason that the proposed scheme reduces the frame collisions by utilizing the spatially orthogonal access scheme, and by minimizing the number of hidden terminals.

\section{CONCLUSIONS}

Future IoT networks are expected to support a massive number of stations/sensors in diverse applications with different QoS requirements. However, the heterogeneous IoT devices operating in ultra-dense network scenarios may be affected by the packet collisions, delays, and low network throughput. In this paper, we proposed a sector-based device grouping scheme for IEEE 802.11ah based IoT networks. In the presented framework, the cloud-center facilitates the grouping process by providing the stations' location information to the AP. The AP forms the sectors, and divides into different groups according to the number of stations and their corresponding locations. In addition, the sector-based grouping allows the substantial improvement on packet collision rate/probability and the throughput of ultra-dense IoT networks by utilizing the spatially orthogonal access mechanism. Via numerical analysis, it has been shown that the proposed sector-based grouping mechanism significantly improves the network throughput and system delay as compared to the conventional DCF and IEEE 802.11ah grouping scheme. In our future work, we plan to implement our proposed mechanism in the IoT cloud platforms to analyze and verify its performance in real world IoT application scenarios, and also extend this work to reduce the mutual interference between different IoT networks for eHealthcare and industry automation applications.

\section{REFERENCES}

[1] J. Lin and et al, "A survey on Internet of Things: Architecture, enabling technologies, security and privacy, and applications," IEEE Internet Things J., vol. 4, no. 5, pp. 1125-1142, Oct 2017.

[2] L. D. Xu, W. He, and S. Li, "Internet of Things in industries: A survey," IEEE Trans. Ind. Informat., vol. 10, no. 4, pp. 2233-2243, Nov 2014.

[3] ITU-R, "Minimum requirements related to technical performance for IMT-2020 radio interface(s)," https://www.itu.int/md/R15-SG05-C0040/en, Feb. 2017, document 5D/TEMP/300(Rev.1).

[4] M. Kamel, W. Hamouda, and A. Youssef, "Ultra-dense networks: A survey," IEEE Commun. Surveys Tuts., vol. 18, no. 4, pp. 2522-2545, Fourthquarter 2016

[5] T. C. Chang, C. H. Lin, K. C. J. Lin, and W. T. Chen, "Load-balanced sensor grouping for IEEE 802.11ah networks," in Proc. IEEE Globecom, Dec 2015, pp. 1-6.

[6] S. K. Sharma and X. Wang, "Live data analytics with collaborative edge and cloud processing in wireless IoT networks," IEEE Access, vol. 5, pp. 4621-4635, 2017

[7] T. Adame and et al, "IEEE 802.11ah: the WiFi approach for M2M communications," IEEE Wireless Commun., vol. 21, no. 6, pp. 144-152, Dec 2014.

[8] N. Nawaz and et al, "Throughput enhancement of restricted access window for uniform grouping scheme in IEEE 802.11ah," in Proc. IEEE ICC, May 2017, pp. 1-7.

[9] E. Khorov, A. Krotov, and A. Lyakhov, "Modelling machine type communication in IEEE 802.11ah networks," in Proc. IEEE ICCW, June 2015, pp. 1149-1154.

[10] L. Tian, J. Famaey, and S. Latre, "Evaluation of the IEEE 802.11ah restricted access window mechanism for dense IoT networks," in Proc IEEE WoWMoM, June 2016, pp. 1-9.

[11] J. O. Seo, C. Nam, S. G. Yoon, and S. Bahk, "Group-based contention in IEEE 802.11ah networks," in Proc. IEEE ICTC, Oct 2014, pp. 709-710.

[12] S. Aust, R. V. Prasad, and I. G. M. M. Niemegeers, "Sector-based RTS/CTS access scheme for high density WLAN sensor networks," in Proc. IEEE LCNW, Sept 2014, pp. 697-701.

[13] S.-G. Yoon, J.-O. Seo, and S. Bahk, "Regrouping algorithm to alleviate the hidden node problem in 802.11ah networks," Comput. Netw., vol. 105, pp. 22-32, 2016

[14] S. Aust, R. V. Prasad, and I. G. M. M. Niemegeers, "IEEE 802.11ah: Advantages in standards and further challenges for sub $1 \mathrm{GHz} W \mathrm{Wi}-\mathrm{Fi}$,' in Proc. IEEE ICC, June 2012, pp. 6885-6889.

[15] L. Zheng and et al, "Performance analysis of group-synchronized DCF for dense IEEE 802.11 networks," IEEE Trans. Wireless Commun., vol. 13, no. 11, pp. 6180-6192, Nov 2014.

[16] P. Sthapit and J.-Y. Pyun, "Station grouping strategy for minimizing association delay in IEEE 802.11ah," IEICE Trans. Commun., vol. E100.B, no. 8, pp. 1419-1427, 2017.

[17] S. Bhandari, S. K. Sharma, and X. Wang, "Cloud-assisted device clustering for lifetime prolongation in wireless IoT networks," in Proc. IEEE CCECE, April 2017, pp. 1-4.

[18] S. K. Sharma and et al, "Physical layer aspects of wireless IoT," in Proc. IEEE ISWCS, Sept 2016, pp. 304-308.

[19] S. Bhandari, S. K. Sharma, and X. Wang, "Latency minimization in wireless IoT using prioritized channel access and data aggregation," in Proc. IEEE Globecom, Dec 2017, pp. 1-6.

[20] P. Chatzimisios, A. C. Boucouvalas, and V. Vitsas, "Packet delay analysis of IEEE 802.11 MAC protocol," Electron. Lett., vol. 39, no. 18, pp. 1358-1359, Sept 2003

[21] G. Bianchi, "Performance analysis of the IEEE 802.11 distributed coordination function," IEEE J. Sel. A. Commun., vol. 18, no. 3, pp. 535-547, March 2000.

[22] Y. Kim and et al, "Optimal throughput analysis of a super dense wireless network with the renewal access protocol," in Proc. IEEE ICCW, June 2015, pp. 2194-2199. 